\title{
Análise Multifractal de Movimentos Persistentes num Sistema de Controle de Atitude ${ }^{1}$
}

A.R. MESQUITA2, K.H. KIENITZ, Divisão de Engenharia Eletrônica, ITA, 12.228-900 São José dos Campos, SP, Brasil.

E.L. REMPEL, Divisão de Ensino Fundamental, ITA, 12.228-900 São José dos Campos, SP, Brasil.

\begin{abstract}
Resumo. O presente trabalho caracteriza movimentos persistentes num sistema de controle de atitude que possui atuadores sujeitos a atrasos e restrições de chaveamento e controlador de primeira ordem, como no caso de satélites ou do VLS (Veículo Lançador de Satélite) brasileiro. Através de simulações, e utilizando o método WTMM (wavelet transform modulus maxima) para calcular o espectro de singularidades dos atratores, foram obtidos diagramas de bifurcação no espaço de parâmetros do controlador $\left(\Re^{2}\right)$, que permitiram discriminar e conhecer os possíveis atratores para cada conjunto de parâmetros. Os resultados foram comparados com a dimensão de Lyapunov.
\end{abstract}

\section{Introdução}

Sistemas de controle de atitude com atuadores com restrições de chaveamento são encontrados em veículos lançadores e satélites $[3,11]$. O projeto de controladores tradicionalmente usado para esses sistemas não explora os atuadores até o seu limite dinâmico. Recentemente foi proposta uma metodologia de projeto que explora melhor o potencial de atuadores com restrições de chaveamento [20]. Em especial verificou-se que, sob certas condições, instalam-se indesejavelmente nesses sistemas movimentos persistentes não-periódicos, eventualmente caóticos.

No trabalho desenvolvido pelo autor em [12], estudos exaustivos dos fenômenos de bifurcação foram realizados para um sistema específico da classe de sistemas de controle de atitude. Nesses estudos, observou-se que, no espaço de parâmetros do controlador, o comportamento dinâmico ótimo do ponto de vista de controle ocorre nas proximidades da fronteira em que surge o referido movimento indesejado. Para esse comportamento ótimo, o ciclo limite alcançou uma amplitude muito abaixo (4\%) daquela especificada em projeto. Esse superdimensionamento do atuador sugere o uso de estratégias de controle mais eficientes. Assim, questionamentos acerca da robustez de um controlador que opere minimizando a amplitude de ciclo-limite, mas que, devido a incertezas de modelagem, é suscetível de instaurar no sistema

\footnotetext{
${ }^{1}$ Esta pesquisa foi apoiada pela Fundação de Amparo à Pesquisa do Estado de São Paulo.

${ }^{2}$ mesquita.alexandre@gmail.com.
} 
um movimento persistente não-periódico, demandaram uma continuação do esforço de pesquisa.

A fim de estudar a robustez desse controlador é importante compreender como ocorrem as transições de um tipo de comportamento para outro. Neste trabalho, os diversos comportamentos do sistema serão entendidos à luz de diagramas de bifurcação que levam em conta a irregularidade de uma função distribuição de probabilidade sobre o atrator. Mais precisamente, essa irregularidade é expressa pelo espectro de singularidades (também chamado multifractal) da função [9], o qual, além de possibilitar discriminar diferentes tipos de comportamento dinâmico, fornece ainda informações sobre a geometria do atrator. A análise aqui proposta é entendida como uma ferramenta alternativa no estudo de sistemas dinâmicos aos quais ferramentas tradicionais podem não ser adequadas. Uma vez que as restrições de acinamento dos atuadores implicam um comportamento dinâmico híbrido no sistema em estudo, o uso de algumas dessas ferramentas tradicionais pode tornar-se oneroso ou mesmo confuso.

Para levantar os diagramas de bifurcação, uma distribuição de probabilidade sobre o atrator foi obtida a partir de respostas simuladas do sistema para cada conjunto de parâmetros. Empregou-se então o método WTMM (wavelet transform modulus maxima), desenvolvido por Muzy et al. [16, 17] com o fim de calcular o espectro multifractal de objetos geométricos. O método tem tido, entre outras, aplicações na análise e discriminação de sinais provenientes de fluxos turbulentos [16], seqüências de DNA [1], demanda de energia elétrica [5] e sinais de ressonância magnética [21].

Na seção 2, apresenta-se o referido método WTMM no contexto do formalismo multifractal. Na seção 3, descreve-se o sistema de controle estudado. Na seção 4, são avaliados os resultados numéricos. Na seção 5, são feitos comentários e considerações finais.

\section{Formalismo Multifractal e o Método WTMM}

Nesta seção comenta-se o formalismo multifractal para sinais unidimensionais introduzido por Muzy et al. [16] e mostra-se o procedimento usado no cálculo do do espectro de singularidades de sinais experimentais a partir do método WTMM. Uma descrição detalhada do método WTMM pode ser encontrada no trabalho de Arneodo et al. [2].

A auto-similaridade é uma propriedade que pode ser verificada em diversos objetos geométricos encontrados na natureza. Em muitos casos um mesmo objeto pode apresentar conjuntos auto-similares com diferentes escalas, as quais podem estar distribuídas num intervalo da reta real. Esses objetos são denominados multifractais. Considerando-se uma trajetória no espaço de fase sobre um conjunto invariante de um sistema dinâmico, é possível definir uma função distribuição de probabilidade nesse espaço. O conjunto de pontos singulares dessa função guarda as características multifractais do atrator em questão.

Dada uma função $S(x)$, define-se $h\left(x_{0}\right)$, seu expoente de Hölder no ponto $x_{0}$, como sendo o maior expoente tal que exista um polinômio $P_{n}$ de grau $n$ satisfazendo 
a relação $\left|S(x)-P_{n}(x)\right| \leq C\left|x-x_{0}\right|^{h\left(x_{0}\right)}$ numa vizinhança de $x_{0}$ com $C$ constante. Sabe-se, então, que $S$ é $n$ vezes diferenciável em $x_{0}$ mas não $n+1$ vezes. O polinômio $P_{n}(x)$ corresponde à série de Taylor de $S(x)$ no ponto $x_{0}$ expandida até a ordem $n$. Assim, $h\left(x_{0}\right)$ mede a "força" da singularidade no ponto $x_{0}$. Quanto maior $h\left(x_{0}\right)$, mais regular é a função nesse ponto, dado que maior será a ordem em que a função é diferenciável no ponto.

Ao analisar a regularidade da função distribuição de probabilidade associada a uma trajetória no espaço de fase, pode-se dividir seu domínio em subconjuntos cujos pontos correspondem a singularidades com expoente de Hölder $h$ e cuja dimensão de Hausdorff é denotada por $D(h)[9]$. O assim chamado espectro de singularidades $D(h)$ está intimamente relacionado às dimensões de Rényi $D(q)$ do atrator [8]. Em especial, $D(q=0)$ corresponde à dimensão de capacidade do objeto, $D(q=1)$, à dimensão de informação [7] e $D(q=2)$, à dimensão de correlação.

Objetos monofractais possuem apenas um ponto em seu espectro de singularidades, ou seja, apenas um fator de escala. Por outro lado, é característica de objetos multifractais um espectro $D(h)$ representado por uma curva de concavidade negativa. O máximo dessa curva coincide com a dimensão de capacidade do objeto. Ainda, quanto mais larga a curva, maior é o intervalo de expoentes que aparecem na função distribuição para o atrator, ou seja, mais complexa é a sua geometria. Neste texto, tal complexidade será analisada a partir da grandeza $\Delta h$, que se define como largura da curva $D(h)$ para metade da máxima dimensão.

O cálculo do espectro multifractal a partir de séries temporais encontra na análise de wavelets uma ferramenta muito eficaz. A transformada de wavelets contínua decompõe uma função em termos de funções elementares que consistem em translações e dilatações de uma mesma função chamada de wavelet-mãe, definida no espaço das funções quadrado-integráveis $[4,14]$. Se essa função possui seus primeiros $n$ momentos nulos, então ela pode ser usada para medir os expoentes de Hölder menores que $n$ [10]. Essa capacidade de medir singularidades de uma função permite usar a transformada de wavelets para calcular o espectro multifractal. No cálculo desse espectro, Muzy et al. [18, 19] mostraram que o método WTMM possibilita uma precisão superior à daquela obtida por outros métodos disponíveis na literatura. As linhas seguintes limitam-se a descrever o algoritmo de análise multifractal usando o método WTMM.

Seja $W_{\psi}[S](x, a)$ a transformada de wavelets contínua da função distribuição de probabilidade $S$, onde a escala $a \in \Re^{+}$, o deslocamento $x \in \Re$ e a wavelet-mãe $\psi(x)$ possui energia unitária. Apoiando-se na dependência $W_{\psi}[S](x, a) \sim a^{h\left(x_{0}\right)+1 / 2}$ discutida em [10], para $x$ numa vizinhança de $x_{0}$, é possível detectar singularidades e extrair seu expoente de Hölder usando a transformada de wavelets. Para isso, o método WTMM define o conjunto de deslocamentos $L(a)$, no qual os coeficientes da transformada apresentam localmente o máximo módulo para uma escala $a$ fixa. Assim, definem-se as seguintes funções:

$$
Z(q, a)=\sum_{x \in L(a)}\left|W_{\psi}[S](x, a)\right|^{q}, q \in \Re
$$




$$
\begin{aligned}
& h(q, a)=\sum_{x \in L(a)} \hat{W}_{\psi}[S](x, a) \ln \left|W_{\psi}[S](x, a)\right|, \\
& D(q, a)=\sum_{x \in L(a)} \hat{W}_{\psi}[S](x, a) \ln \left|\hat{W}_{\psi}[S](x, a)\right|,
\end{aligned}
$$

onde $\hat{W}_{\psi}[S](x, a)=\left|W_{\psi}[S](x, a)\right| / Z(q, a)$. Dessa forma, obtém-se o espectro de singularidades $D(h)$ de $S$ combinando os resultados abaixo:

$$
\begin{gathered}
h(q)=\lim _{a \rightarrow 0^{+}} \frac{h(q, a)}{\ln a}-\frac{1}{2}, \\
D(q)=\lim _{a \rightarrow 0^{+}} \frac{D(q, a)}{\ln a} .
\end{gathered}
$$

É importante notar que aqui se fez uma alteração significativa do método original. Neste, os deslocamentos $L(a)$ são conectados definindo-se curvas parametrizadas em $a$. As funções de partição $Z(q, a), h(q, a)$ e $D(q, a)$ são definidas em função do supremo ao longo dessas linhas para as escalas menores que $a$. A simplificação aqui adotada destina-se à redução do tempo computacional, uma vez que determinar as linhas de máximo módulo é tarefa que requer grande esforço computacional e deverá ser aplicada a um grande número de pontos no espaço de parâmetros do controlador. Em contrapartida, essa simplificação afeta a precisão do cálculo do espectro de singularidades quando $q<0$. Testes preliminares mostraram tolerável essa perda de precisão.

\section{O Sistema de Controle de Rolamento do VLS}

Nesta seção, o sistema de interesse é apresentado sob a perspectiva da engenharia de controle. Leitores pouco familiarizados com conceitos como função de transferência e diagramas de blocos podem recorrer a textos introdutórios como [6]. O problema aqui discutido trata especificamente do controle de rolamento do veículo lançador de satélites brasileiro. Uma formulação detalhada poderá ser encontrada em [12]. Considere-se um corpo rígido como um satélite ou um veículo lançador, cuja atitude $\phi(t)$ deva ser controlada por um par de atuadores, conforme ilustrado na figura 1.

Tal controle visa a manter reguladas posição e velocidade angular de rolamento, as quais sofrem influência de perturbações internas e externas. A importância de limitar a velocidade angular de rolamento está em reduzir o acoplamento dos modos longitudinal e látero-direcionais.

É dado um momento de inércia longitudinal $J=1500\left[\mathrm{Kgm}^{2}\right]$. O sistema de atuação consiste em quatro motores com funcionamento "on-off" e com fortes restrições de acionamento, separados por um intervalo angular de $180^{\circ}$, acionados aos pares de forma diferencial para que cada par atue com um momento num sentido. As características desses atuadores são as seguintes: 


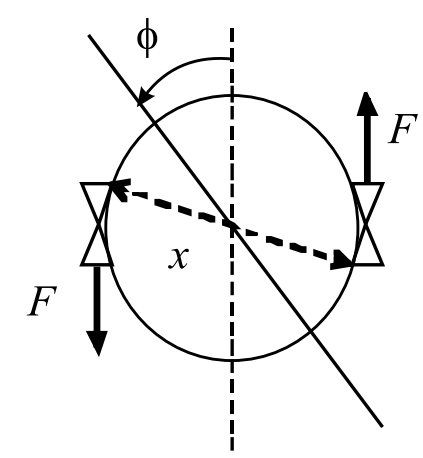

Figura 1: Veículo com dois pares de motores a propulsão

- Empuxo de cada motor: $F=400 \pm 20[\mathrm{~N}]$

- Braço do binário: $x=0.77[\mathrm{~m}]$

- Atraso modelado pela função de transferência $G(s)=\frac{86.8^{2}}{(s+86.8)^{2}}$

- Restrições de acionamento:

- duração mínima de cada pulso: 100 [ms]

- intervalo de tempo mínimo entre o desligamento e o acionamento do mesmo par: $50[\mathrm{~ms}]$

- intervalo de tempo mínimo entre o desligamento de um par e o acionamento do par oposto: 500 [ms]

Para se atingirem os requisitos de desempenho, o sistema é realimentado com os valores de posição e velocidade angular. Os parâmetros para análise serão o zero $(z)$ e o pólo $(p)$ do controlador linear de primeira ordem:

$$
C(s)=\frac{s-z}{s-p} .
$$

Uma visão completa do diagrama de blocos do sistema controlado é apresentada na figura 2.

\section{Cálculo do Espectro de Singularidades}

A programação para estimação do espectro de singularidades se realizou em Matlab. O sistema de controle foi simulado utilizando-se a ferramenta xPC Target do Matlab. Munindo-se de dois PCs, essa ferramenta permite implementar uma configuração de hardware mestre-escravo capaz de realizar simulações com alta velocidade, oferecendo a baixos custos as mesmas funcionalidades de uma placa de DSP de alto desempenho. 


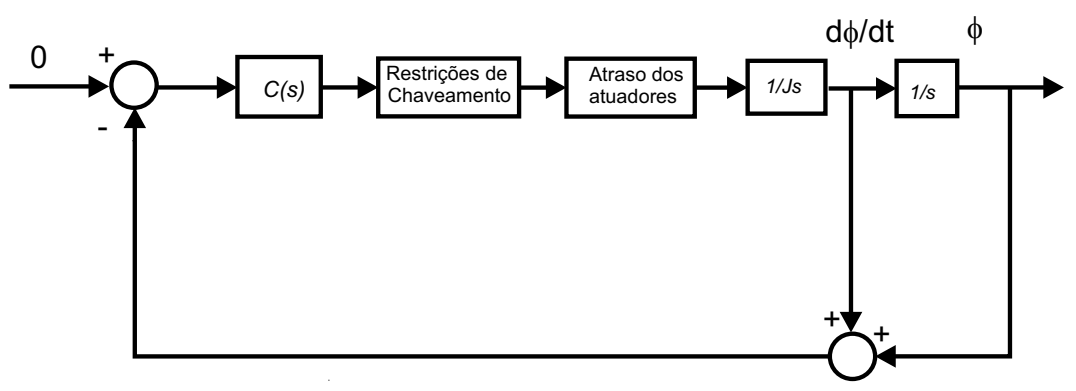

Figura 2: Diagrama de blocos do sistema controlado

A partir de uma mesma condição inicial e variando-se os parâmetros $p$ e $z$ numa grade de 600 pontos, levantou-se a resposta $\phi(t)$ do sistema para um horizonte de simulação de $600[\mathrm{~s}]$, sendo descartados os $24[\mathrm{~s}]$ iniciais correspondentes à resposta transitória. Utilizou-se passo de integração de 2 [ms] e método de integração explícito de $4^{a}$ ordem. Calculou-se o espectro de singularidades da distribuição de probabilidade sobre o conjunto de máximos locais $\phi_{\max }(k)$ de $\phi(t)$. Para se obter tal distribuição, calculou-se a freqüência de $\phi_{\max }(k)$ sobre o intervalo $\left[\min \left\{\phi_{\max }(k)\right\}-0.005, x\right]$, variando-se $x$ com um passo mínimo de $10^{-4}$ no intervalo $\left[\min \left\{\phi_{\max }(k)\right\}-0.005, \max \left\{\phi_{\max }(k)\right\}+0.005\right]$. O número de pontos $N$ nesse intervalo foi limitado a 4096. A wavelet adotada foi aquela conhecida como chapéu mexicano, que consiste no oposto da segunda derivada da função gaussiana, a qual possui os dois primeiros momentos nulos. Portanto, é capaz de determinar o expoente de Hölder de singularidades com valor menor que 2. Consideraram-se escalas de 2 até $N / 32$, distribuindo-se 12 vozes em cada oitava. Adotou-se $q$ uniformemente distribuído em $[-8,8]$. Para se avaliarem os resultados obtidos, foi calculada a dimensão de Lyapunov para o sistema empregando-se a técnica proposta em [15], que permite calcular esses expoentes mesmo para sistemas com descontinuidades de fluxo.

A figura 3 exibe a dimensão de informação da mencionada projeção do atrator resultante de cada combinação dos parâmetros $p$ e $z$ e calculada usando o método WTMM. A figura 4 mostra a dimensão de Lyapunov para a mesma grade de parâmetros. De modo geral, o espectro de Lyapunov mostra que os três patamares correspondem, por ordem crescente de dimensão, a movimentos periódico, quaseperiódico-similar (QPS) e caótico. Uma descrição detalhada desses movimentos é feita em [13].

A notável correlação entre os gráficos sugere a verossimilhança das estimativas efetuadas pelo método WTMM. Sobretudo, o método permite distinguir com clareza os pontos de bifurcação, onde ocorrem mudanças bruscas nos valores de $D_{I}$. Sabendo-se que dimensões próximas a zero caracterizam a interseção de um atrator periódico com um hiperplano, observa-se que o primeiro gráfico possibilita uma clara distinção desse tipo de comportamento. Contudo, para altos valores de $z$, o 


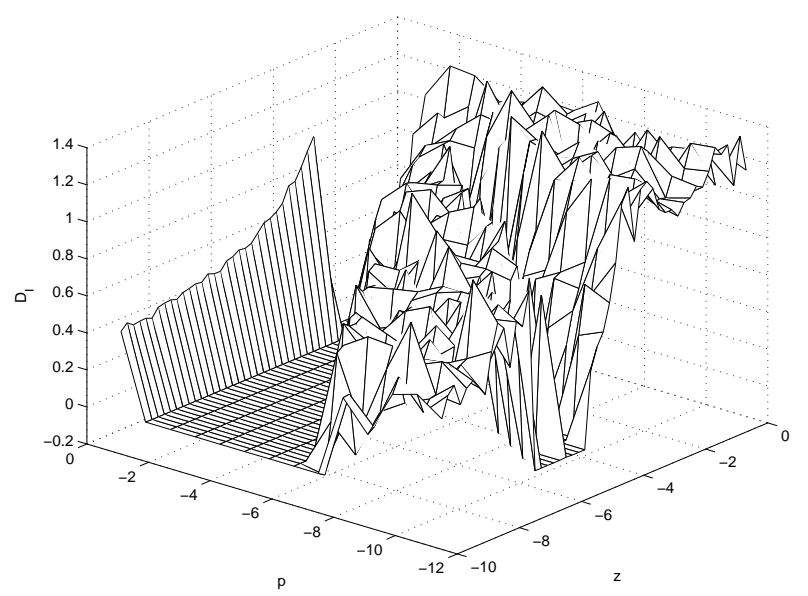

Figura 3: Diagrama biparamétrico para a estimativa da dimensão de informação

gráfico 3 não permite distinguir com a mesma clareza um comportamento caótico de um QPS. Deve-se alertar, porém, para o fato de que não é possível comparar diretamente a dimensão de informação com a dimensão de Lyapunov, pois esta diz respeito a todo o atrator, ao passo que a primeira se calculou sobre uma projeção de uma intersecção do atrator com um hiperplano. Essa é uma explicação plausível de por que o primeiro gráfico aparenta ser uma degeneração do segundo.

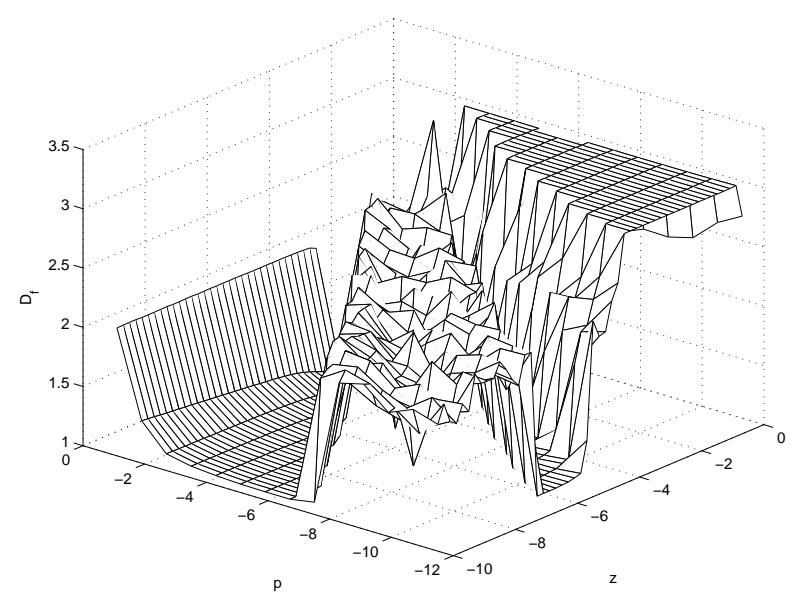

Figura 4: Diagrama biparamétrico para a dimensão de Lyapunov

A análise multifractal provê outras grandezas que também contribuem para a caracterização dos atratores. Uma delas é o expoente de Hölder mais freqüente $h_{\max }=h(q=0)$. Cálculos do expoente de Hölder mostraram que o atrator caótico 
apresenta irregularidade levemente maior que a do QPS. Observou-se uma distribuição de probabilidade descontínua $(h<1)$ no primeiro e contínua $(h>1)$ no último.

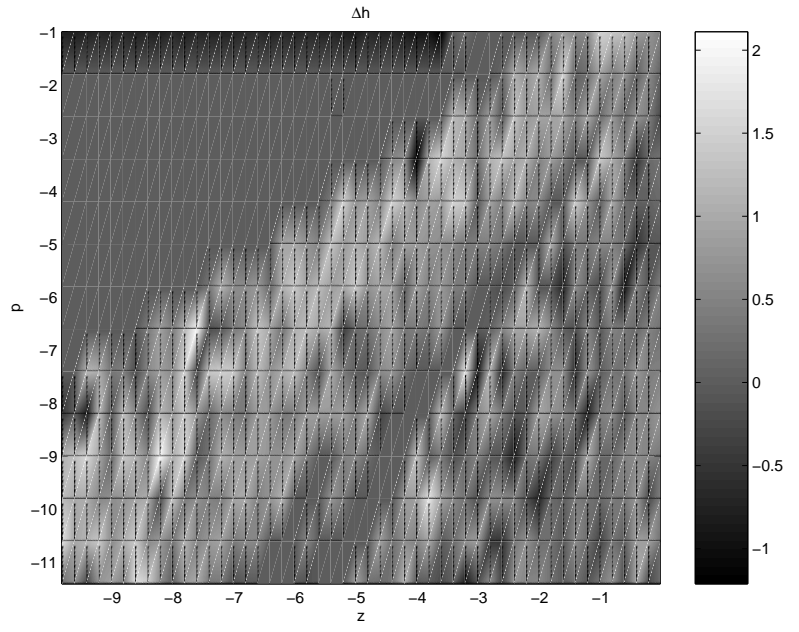

Figura 5: Diagrama biparamétrico para $\Delta h$

Outra grandeza é a largura $\Delta h$ que é exibida na figura 5 e mostra que tanto o movimento QPS quanto o caótico exibem atratores multifractais. Contudo, para grande parte dos casos, o movimento QPS apresenta um espectro de singularidades mais largo. Tal complexidade geométrica que se tem observado no atrator QPS está associada à natureza dinâmica híbrida que a lógica de chaveamento introduz no sistema. Essa lógica faz com que o atrator aparente dimensão 3 quando observado por intervalos curtos e dimensão 2 ao ser observado por um longo tempo [13]. Essa variedade de escalas, que está associada à presença de estados discretos no sistema, fica bem retratada por essa maior largura do espectro multifractal.

\section{Conclusão}

O trabalho descrito neste texto ocupou-se da confecção de diagramas de bifurcação que exibem grandezas relacionadas ao espectro multifractal de possíveis atratores num sistema de controle de atitude. Tendo sido comparados com a dimensão de Lyapunov, os resultados fornecem sinais favoráveis para o uso do espectro multifractal como ferramenta de discriminação de comportamento dinâmico. Os diagramas obtidos exibem as bifurcações de maneira clara. Ademais, provêem conhecimento sobre a irregularidade geométrica dos atratores, o que é de interesse teórico para o estudo da geometria fractal em sistemas dinâmicos.

Embora a técnica empregada seja computacionalmente inferior à dos expoentes de Lyapunov, ela se apresenta como uma interessante alternativa em casos em que o espectro de Lyapunov não pode ser facilmente calculado, como aqueles em que não se dispõe de um modelo dinâmico satisfatório. No caso de sistemas híbridos com 
um grande número de estados discretos essa técnica se faria bastante interessante, uma vez que o cálculo dos expoentes de Lyapunov seria trabalhoso e de demorada convergência. Outra aplicação promissora se encontra no estudo de séries temporais ruidosas, uma vez que o método fornece uma análise mais precisa que algoritmos tradicionais de cálculo de dimensão.

\begin{abstract}
This work presents results concerning the singularity spectrum of attractors arising from attitude control systems with time-constrained delayed actuators and first order controller, such as those found in satellite launchers or satellites. Via simulation of the system and employing WTMM (wavelet transform modulus maxima) method, we have been able to build bifurcation diagrams that allowed to gain knowledge of possible attractors and to discriminate them.
\end{abstract}

\title{
Referências
}

[1] A. Arneodo et al. Wavelet based fractal analysis of DNA sequences, Physica D 96 (1996), 291-320.

[2] A. Arneodo et al., The thermodynamics of fractals revisited with wavelets, Physica A, 213 (1995), 232-275.

[3] G. Avanzini, Bifurcation analysis of attitude dynamics in rigid spacecraft with switching control logics, J. Guid., Control, Dynamics, 24 (2001), 953-959.

[4] I. Daubechies, "Ten Lectures on Wavelets", SIAM, Philadelphia, 1992.

[5] M. Degaudenzi, C. Arizmendi, Wavelet-based fractal analysis of electrical power demand, Fractals 8 (2000), 239-245.

[6] G. F. Franklin, J.D. Powell, A. Emami-Naeini, "Feedback Control of Dynamic Systems", Addison Wesley, Reading, 1994.

[7] P. Frederickson et al., The liapunov dimension of strange attractors, Journal of Differential Equations, 49 (1983), 185-207.

[8] P. Grassberger, I. Procaccia, Dimensions and entropies of strange attractors from a fluctuating dynamics approach, Physica D, 13 (1984), 34-54.

[9] T. Halsey et al., Fractal measures and their singularities: the characterization of strange sets, Physical Review A, 33 (1986), 1141-1151.

[10] S. Mallat, W. Hwang, Singularity detection and processing with wavelets, IEEE Trans. on Information Theory, 38 (1992), 617-643.

[11] J. Mendel, On-off limit-cycle controllers for reaction-jet-controlled systems, IEEE Transactions on Automatic Control, 15 (1970), 285-299.

[12] A. Mesquita, "Análise Paramétrica de um Sistema de Controle de Atitude com Atuadores Sujeitos a Restrições de Chaveamento", Trabalho de Graduação. IEE, ITA, São José dos Campos, SP, 2004. Disponível em www.bibl.ita.br. 
[13] A. Mesquita, "Caracterização e análise de robustez do comportamento dinâmico de um sistema de controle de atitude com atuadores sujeitos a restrições de chaveamento e controlador de primeira ordem", Tese de Mestrado. IEE, ITA, São José dos Campos, SP, 2006. Disponível em www.bibl.ita.br.

[14] Y. Meyer, S. Roques, eds., "Progress in Wavelet Analysis and Applications", Frontières, Gif sur Yvette, 1993.

[15] P. Müller, Calculation of lyapunov exponents for dynamic systems with discontinuities, Chaos, Solitons $\&$ Fractals, 5 (1995), 1671-1681.

[16] J. Muzy et al., Wavelets and multifractal formalism for singular signals: application to turbulence data. Phys. Rev. Lett., 67 (1991), 3515-3518.

[17] J. Muzy et al., The multifractal formalism revisited with wavelets, Int. J. Bifurc. Chaos, 4 (1993), 245- 303.

[18] J. Muzy et al., Multifractal formalism for fractal signals, The structure function method versus the wavelet-transform modulus maxima method, Phys. Rev. E, 47 (1993), 875-884.

[19] J. Muzy et al., Multifractal Formalism for Singular Signals based on Wavelet Analysis, em "Progress in wavelet analysis and applications" (Yves e Meyers, eds.) pp. 323-336, Frontiers, Gif sur Yvette, 1993.

[20] N. Oliveira, K. Kienitz, Attitude controller design for a system using actuators with switching-time restrictions and delays, "AIAA Guidance, Navigation, and Control Conference", Denver, 2000.

[21] Y. Shimizu et al., Wavelet-based multifractal analysis of fMRI time series. NeuroImage, 22 (2004), 1195-1202. 